\title{
Finite-Time Synchronizing Fractional-Order Chaotic Volta System with Nonidentical Orders
}

\author{
Jian-Bing Hu and Ling-Dong Zhao \\ School of Electronics and Information, Nantong University, Nantong, Jiangsu 226019, China \\ Correspondence should be addressed to Ling-Dong Zhao; zhaolingdong@163.com
}

Received 3 October 2012; Accepted 27 January 2013

Academic Editor: Engang Tian

Copyright ( 2013 J.-B. Hu and L.-D. Zhao. This is an open access article distributed under the Creative Commons Attribution License, which permits unrestricted use, distribution, and reproduction in any medium, provided the original work is properly cited.

We investigate synchronizing fractional-order Volta chaotic systems with nonidentical orders in finite time. Firstly, the fractional chaotic system with the same structure and different orders is changed to the chaotic systems with identical orders and different structure according to the property of fractional differentiation. Secondly, based on the lemmas of fractional calculus, a controller is designed according to the changed fractional chaotic system to synchronize fractional chaotic with nonidentical order in finite time. Numerical simulations are performed to demonstrate the effectiveness of the method.

\section{Introduction}

Fractional calculus, starting from some speculations of G. W. Leibniz $(1695,1697)$ and L. Euler $(1730)$, has a history of over 300 years old. But its application to physics and engineering has attracted lots of attention only in the recent decades. Up until now, people have investigated and developed many methods to synchronize fractional dynamical systems with identical order, for example, one-way coupling method [1], washout filter control [2], sliding mode [3], fuzzy control $[4,5]$, pinning control [6], active control [7], adaptive control [8-10], time-varying delays control [11-13], further fruits can be found in [14-16], and so forth.

In general, the order of the drive fractional chaotic system may not be equal with the order of the response fractional chaotic system. It is necessary to study synchronizing fractional chaotic system with nonidentical order. In [9], based on the stability theorem of linear fractional order systems and Laplace transform theory, synchronizing nonidentical chaotic fractional order systems is realized. But the controller designing schedule is intricate, and the process is also difficult to understand. In [10], the authors utilize the integral property of fractional differential function to synchronize fractional chaotic system with nonidentical order. But the authors neglect the result of fractional integration concerning the initial value condition, and a different result can be gotten under different initial value condition by integration, then the approach cannot be effectively broaden.

Generally, synchronizing error systems converge to zero in infinite time. But in some cases such as communication, the synchronizing errors are usually demanded to converge to zero in finite time. Finite-time synchronizing integerorder chaotic has been studied $[17,18]$. To the best of our knowledge, synchronizing fractional-order in finite time has rarely been reported.

Motivated by the above discussion, we propose a new approach to design a controller to realize finite-time synchronizing fractional order chaotic system with nonidentical orders based on the properties of fractional differentiation. The theorem is easy to understand. Numerical simulations are used to verify the effectiveness of this approach.

The rest of the paper is organized as follows. In Section 2, primary definitions and lemma about fractional-order differentiation are introduced. In Section 3, based on the lemmas of fractional calculus, a controller is designed to realize finitetime synchronizing fractional-order chaotic Volta system with nonidentical orders. Also, numerical simulation results are presented in Section 4 to show the effectiveness of the designed controller. Finally, the conclusion is made in Section 5 . 


\section{Preliminaries}

There are three commonly used definitions of the fractionalorder differential operator: Grunwald-Letnikov, RiemannLiouville, and Caputo definitions. In this paper, we study the stability of fractional system based on the Caputo definition.

Definition 1. The Caputo definition of fractional order can be expressed as [19]

$$
{ }_{a}^{C} D_{t}^{\alpha} f(t)=\frac{1}{\Gamma(n-\alpha)} \times \int_{a}^{t}(t-\tau)^{-\alpha+n-1} f^{(n)}(\tau) d \tau,
$$

where $n$ is the first integer which is not less than $\alpha$, for example, when $0<\alpha \leq 1, n=1$, and $\Gamma(\cdot)$ is gamma function.

Lemma 2 (see [10]). Suppose $f(t) \in C_{a}^{\alpha}([a, b]), D_{a}^{\alpha} f(t) \in$ $C_{a}^{\beta}([a, b]), \alpha>0, \beta>0, m-1<\beta<m$, and $n-1<\alpha<n$, then:

$$
{ }_{a}^{C} D_{t}^{\beta}\left({ }_{a}^{C} D_{t}^{\alpha} f(t)\right)={ }_{a}^{C} D_{t}^{\alpha}\left({ }_{a}^{C} D_{t}^{\beta} f(t)\right)={ }_{a}^{C} D_{t}^{\alpha+\beta} f(t) .
$$

Lemma 3 (see [20]). Consider

$$
{ }_{a}^{C} D_{t}^{\alpha} x^{u}=\frac{\Gamma(u+1)}{\Gamma(u+1-\alpha)} x{ }_{a}^{u-\alpha} D_{t}^{\alpha} x
$$

Lemma 4 (see [21]). For $a, b>0,0<c<1(a, b, c \in R)$ the following inequality holds:

$$
(a+b)^{c} \leq a^{c}+b^{c} .
$$

\section{Finite-Time Synchronizing Nonidentical Orders Fractional Chaotic Volta System}

In this section, we investigate finite-time synchronizing fractional-order chaotic Volta systems with nonidentical orders.

The fractional-order Volta's system is depicted by

$$
\begin{gathered}
D^{\alpha} x_{1}(t)=-x_{1}-a x_{2}-x_{3} x_{2}, \\
D^{\alpha} x_{2}(t)=-x_{2}-b x_{1}-x_{1} x_{3}, \\
D^{\alpha} x_{3}(t)=c x_{3}+x_{1} x_{2}+1 .
\end{gathered}
$$

When system parameters $(a, b, c)=(19,11,0.73)$ and order $\alpha>0.97$, the system (5) has chaotic attractor [22].

Define the system (5) as the drive system, and the response system is given as follows:

$$
\begin{gathered}
D^{\beta} y_{1}(t)=-y_{1}-a y_{2}-y_{3} y_{2}+u_{1}(t), \\
D^{\beta} y_{2}(t)=-y_{2}-b y_{1}-y_{1} y_{3}+u_{2}(t), \\
D^{\beta} y_{3}(t)=c y_{3}+y_{1} y_{2}+1+u_{3}(t),
\end{gathered}
$$

where $\beta>\alpha$ and $u(t)=\left(u_{1}(t), u_{2}(t), u_{3}(t)\right)$ is the nonlinear controller to be designed.
According to Lemma 2 , taking $(\beta-\alpha)$ derivative of both sides of (5), we get

$$
\begin{gathered}
D^{\beta} x_{1}(t)=D^{\beta-\alpha}\left(D^{\alpha} x_{1}(t)\right)=D^{\beta-\alpha}\left(-x_{1}-a x_{2}-x_{3} x_{2}\right), \\
D^{\beta} x_{2}(t)=D^{\beta-\alpha}\left(D^{\alpha} x_{2}(t)\right)=D^{\beta-\alpha}\left(-x_{2}-b x_{1}-x_{1} x_{3}\right), \\
D^{\beta} x_{3}(t)=D^{\beta-\alpha}\left(D^{\alpha} x_{3}(t)\right)=D^{\beta-\alpha}\left(c x_{3}+x_{1} x_{2}+1\right) .
\end{gathered}
$$

Thus, the synchronizing system (5) and the system (6) with nonidentical orders are transformed into the synchronizing system (6) and system (7) with identical orders,

Define the error variables as

$$
\begin{aligned}
& e_{1}=y_{1}-x_{1}, \\
& e_{2}=y_{2}-x_{2}, \\
& e_{3}=y_{3}-x_{3} .
\end{aligned}
$$

Subtracting (7) from (6), we obtain the error dynamics

$$
\begin{aligned}
D^{\beta} e_{1}(t)= & -y_{1}-a y_{2}-y_{3} y_{2}+u_{1}(t) \\
& -D^{\beta-\alpha}\left(-x_{1}-a x_{2}-x_{3} x_{2}\right), \\
D^{\beta} e_{2}(t)= & -y_{2}-b y_{1}-y_{1} y_{3}+u_{2}(t) \\
& -D^{\beta-\alpha}\left(-x_{2}-b x_{1}-x_{1} x_{3}\right), \\
D^{\beta} e_{3}(t)= & c y_{3}+y_{1} y_{2}+1+u_{3}(t) \\
& -D^{\beta-\alpha}\left(c x_{3}+x_{1} x_{2}+1\right) .
\end{aligned}
$$

The key problem is how to design the controller $u(t)$ to realize chaotic synchronization in finite time.

Theorem 5. If the controller satisfies

$$
\begin{aligned}
u_{1}(t)= & D^{\beta-\alpha}\left(-x_{1}-a x_{2}-x_{3} x_{2}\right)-\left(-x_{1}-a x_{2}-x_{3} x_{2}\right) \\
& +\left(a+x_{3}+b+y_{3}\right) e_{2}-k e_{1}^{r}, \\
u_{2}(t)= & D^{\beta-\alpha}\left(-x_{2}-b x_{1}-x_{1} x_{3}\right) \\
& -\left(-x_{2}-b x_{1}-x_{1} x_{3}\right)-k e_{2}^{r}, \\
u_{3}(t)= & D^{\beta-\alpha}\left(c x_{3}+x_{1} x_{2}\right) \\
& -\left(c x_{3}+x_{1} x_{2}+1\right)-\lambda e_{3}-k e_{3}^{r},
\end{aligned}
$$

where $-1<r<1, k>0$, and $\lambda>c$, the response system (6) will synchronize with the derive system (7) in the finite time $T=\left(v^{\beta-(1+r) / 2}(0)(\Gamma(2) / k \Gamma(2+\beta))(\Gamma((1-r) / 2) \Gamma(1+\beta) / \Gamma(\beta+\right.$ $(1-r) / 2)))^{1 / \beta}$. 

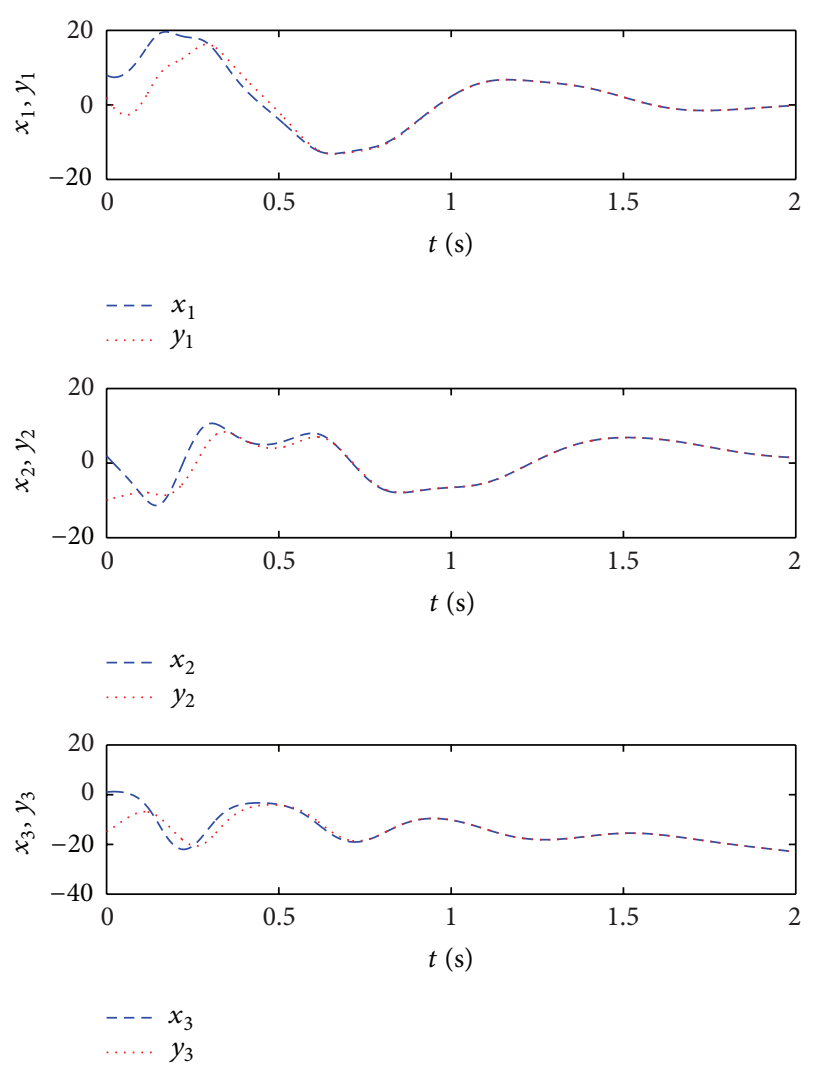

FIgURE 1: Time responses of state variables in the response system and the drive system.

Proof. Substituting (10) into (9), we get

$$
\begin{aligned}
& {\left[\begin{array}{l}
D^{\beta} e_{1}(t) \\
D^{\beta} e_{2}(t) \\
D^{\beta} e_{3}(t)
\end{array}\right]} \\
& \quad=\left[\begin{array}{ccc}
-1-k e_{1}^{r-1} & b+y_{3} & -y_{2} \\
-b-y_{3} & -1-k e_{2}^{r-1} & -x_{1} \\
y_{2} & x_{1} & c-\lambda-k e_{3}^{r-1}
\end{array}\right]\left[\begin{array}{l}
e_{1} \\
e_{2} \\
e_{3}
\end{array}\right] .
\end{aligned}
$$

Then

$$
\begin{aligned}
{\left[\begin{array}{lll}
e_{1} & e_{2} & e_{3}
\end{array}\right]\left[\begin{array}{c}
D^{\beta} e_{1}(t) \\
D^{\beta} e_{2}(t) \\
D^{\beta} e_{3}(t)
\end{array}\right] } \\
=e_{1} D^{\beta} e_{1}(t)+e_{2} D^{\beta} e_{2}(t)+e_{3} D^{\beta} e_{3}(t) \\
=\left(-1-k e_{1}^{r-1}\right) e_{1}^{2}(t)+\left(-1-k e_{2}^{r-1}\right) e_{2}^{2}(t) \\
+\left(c-\lambda-k e_{3}^{r-1}\right) e_{3}^{2}(t) \\
\leq-k e_{1}^{r+1}-k e_{2}^{r+1}-k e_{3}^{r+1} \\
\leq-k\left(e_{1}^{2}+e_{2}^{2}+e_{3}^{2}\right)^{(1+r) / 2} .
\end{aligned}
$$

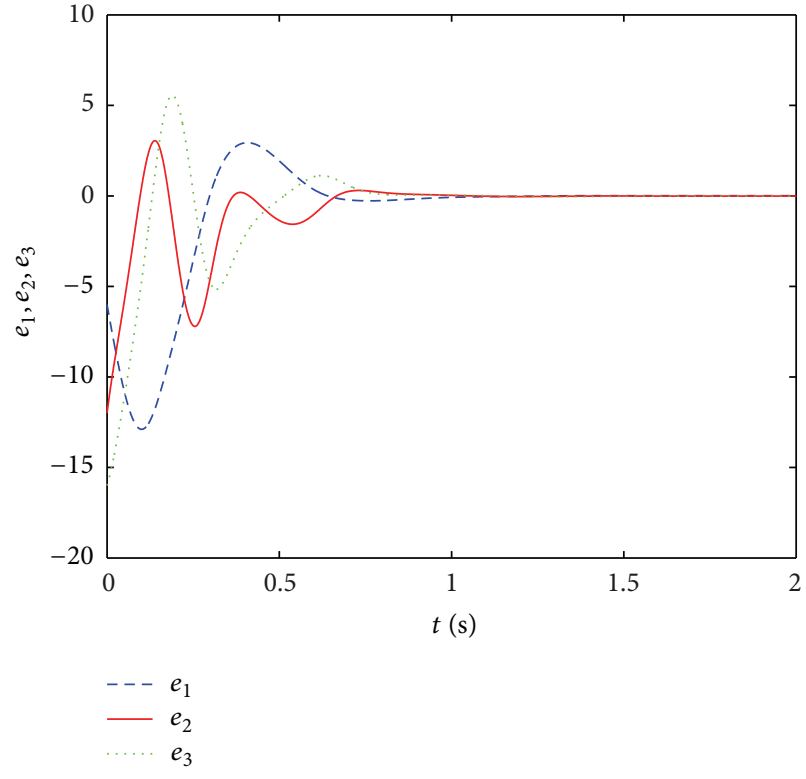

FIGURE 2: Synchronization errors $e_{1}(t), e_{2}(t)$, and $e_{3}(t)$ with time $t$.

Using Lemma 3, we have

$$
\begin{aligned}
& e_{1} D^{\beta} e_{1}(t)+e_{2} D^{\beta} e_{2}(t)+e_{3} D^{\beta} e_{3}(t) \\
& \quad=\frac{\Gamma(2)}{\Gamma(2+\beta)} D^{\beta}\left(e_{1}^{2}+e_{2}^{2}+e_{3}^{2}\right)^{(1+\beta) / 2} .
\end{aligned}
$$

Define $v=e_{1}^{2}+e_{2}^{2}+e_{3}^{2}$, and after some simple manipulations, we get

$$
\frac{\Gamma(2)}{\Gamma(2+\beta)} D^{\beta} v^{(1+\beta) / 2} \leq-k v^{(1+r) / 2} .
$$

Then

$$
v^{(-1-r) / 2} D^{\beta} v^{(1+\beta) / 2} \leq-\frac{\Gamma(2+\beta)}{\Gamma(2)} k,
$$

and based on Lemma 3, one gets

$$
D^{\beta} v^{\beta-(1+r) / 2} \leq-k \frac{\Gamma(2+\beta)}{\Gamma(2)} \frac{\Gamma(\beta+(1-r) / 2)}{\Gamma((1-r) / 2)} .
$$

Taking integral of both sides of (16) from 0 to $T$,

$$
\begin{aligned}
& v^{\beta-(1+r) / 2}(T)-v^{\beta-(1+r) / 2}(0) \\
& \quad \leq-k \frac{\Gamma(2+\beta)}{\Gamma(2)} \frac{\Gamma(\beta+(1-r) / 2)}{\Gamma((1-r) / 2)} \frac{1}{\Gamma(1+\beta)} T^{\beta} .
\end{aligned}
$$

Then when

$$
T \leq\left(v^{\beta-(1+r) / 2}(0) \frac{\Gamma(2)}{k \Gamma(2+\beta)} \frac{\Gamma((1-r) / 2) \Gamma(1+\beta)}{\Gamma(\beta+(1-r) / 2)}\right)^{1 / \beta},
$$

$v(T)=0$, that is to say, the synchronization is realized in finite time $T=\left(v^{\beta-(1+r) / 2}(0)(\Gamma(2) / k \Gamma(2+\beta))(\Gamma((1-r) / 2) \Gamma(1+\right.$ $\beta) / \Gamma(\beta+(1-r) / 2)))^{1 / \beta}$. The proof is completed. 


\section{Numerical Simulations}

In this section, the simulation results are carried out to show the effectiveness of the designed controller.

Take $\lambda=5, k=3$, and $r=0.8$ and the order of the drive system as $\alpha=0.975$ and the order of the response system as $\beta=0.98$; the initial values are selected as $\left(x_{1}(0), x_{2}(0), x_{3}(0)\right)=(8,2,1)$ and $\left(y_{1}(0), y_{2}(0), y_{3}(0)\right)=$ $(2,-10,-15)$. The numerical simulations are shown as Figures 1 and 2. We can see that the synchronization is achieved in finite time from the simulation result. The response system synchronizes with the drive system in finite time.

\section{Conclusion}

In this paper, we study the finite-time synchronization of fractional chaotic systems with nonidentical orders. A new approach is proposed to design the controller. The approach is not only simple but also easy to understand, which can broad the approach of synchronizing fractional chaotic system. Numerical simulations show the effectiveness of the approach.

\section{References}

[1] X. J. Wu, J. Li, and G. R. Chen, "Chaos in the fractional order unified system and its synchronization," Journal of the Franklin Institute, vol. 345, no. 4, pp. 392-401, 2008.

[2] S. B. Zhou, X. R. Lin, and H. Li, "Chaotic synchronization of a fractional-order system based on washout filter control," Communications in Nonlinear Science and Numerical Simulation, vol. 16, no. 3, pp. 1533-1540, 2011.

[3] S. Dadras and H. R. Momeni, "Control of a fractional-order economical system via sliding mode," Physica A, vol. 389, no. 12, pp. 2434-2442, 2010.

[4] E. Tian, D. Yue, T. C. Yang, Z. Gu, and G. Lu, "TS fuzzy modelbased robust stabilization for networked control systems with probabilistic sensor and actuator failure," IEEE Transactions on Fuzzy Systems, vol. 19, no. 3, pp. 553-561, 2011.

[5] Y. A. Zheng, Y. B. Nian, and D. J. Wang, "Controlling fractional order chaotic systems based on Takagi-Sugeno fuzzy model and adaptive adjustment mechanism," Physics Letters A, vol. 375, pp. 125-129, 2010.

[6] Y. Tang, H. Gao, J. Kurths, and J. Fang, "Evolutionary pinning control and its application in UAV coordination," IEEE Transactions on Industrial Informatics, vol. 8, no. 4, pp. 828-838, 2012.

[7] S. Bhalekar and V. Daftardar-Gejji, "Synchronization of different fractional order chaotic systems using active control," Communications in Nonlinear Science and Numerical Simulation, vol. 15, no. 11, pp. 3536-3546, 2010.

[8] W. Zhu, J. Fang, and Y. Tang, "Identification of fractional-order systems via a switching differential evolution subject to noise perturbations," Physics Letters A, vol. 376, no. 45, pp. 3113-3120, 2012.

[9] Z. M. Odibat, "Adaptive feedback control and synchronization of non-identical chaotic fractional order systems," Nonlinear Dynamics, vol. 60, no. 4, pp. 479-487, 2010.

[10] G. Q. Si, Z. Y. Sun, Y. B. Zhang, and W. Q. Chen, "Projective synchronization of different fractional-order chaotic systems with non-identical orders," Nonlinear Analysis: RealWorld Applications, vol. 13, no. 4, pp. 1761-1771, 2012.
[11] H. Li, Z. J. Ning, and Y. H. Yin, "Synchronization and state estimation for singular complex dynamical networks with timevarying delays," Communications in Nonlinear Science and Numerical Simulation, vol. 18, no. 1, pp. 194-208, 2013.

[12] H. Li, "Cluster synchronization stability for stochastic complex dynamical networks with probabilistic interval time-varying delays," Journal of Physics A, vol. 44, no. 10, Article ID 105101, 2011.

[13] H. Li, W. K. Wong, and Y. Tang, "Global synchronization stability for stochastic complex dynamical networks with probabilistic interval time-varying delays," Journal of Optimization Theory and Applications, vol. 152, no. 2, pp. 496-516, 2012.

[14] E. Tian, D. Yue, and C. Peng, "Brief Paper: reliable control for networked control systems with probabilistic sensors and actuators faults," IET Control Theory and Applications, vol. 4, no. 8, pp. 1478-1488, 2010.

[15] Y. Tang, W. Zou, J. Lu, and J. Kurths, "Stochastic resonance in an ensemble of bistable systems under stable distribution noises and nonhomogeneous coupling," Physical Review E, vol. 85, no. 4, Article ID 046207, 6 pages, 2012.

[16] Y. Tang, Z. Wang, H. Gao, S. Swift, and J. Kurths, "A constrained evolutionary computation method for detecting controlling regions of cortical networks," IEEE/ACM Transactions on Computational Biology and Bioinformatics, vol. 9, no. 6, pp. 15691581, 2012.

[17] H. Wang, Z.-Z. Han, Q.-Y. Xie, and W. Zhang, "Finite-time synchronization of uncertain unified chaotic systems based on CLF," Nonlinear Analysis: Real World Applications, vol. 10, no. 5, pp. 2842-2849, 2009.

[18] H. Wang and Z. Z. Han, "Finite-time chaos synchronization of unified chaotic system with uncertain parameters," Communications in Nonlinear Science and Numerical Simulation, vol. 14, no. 5, pp. 2239-2247, 2009.

[19] M. Caputo, "Linear models of dissipation whose $Q$ is almost frequency independent-II," Journal of the Royal Society of Western Australia, vol. 13, no. 5, pp. 529-539, 1967.

[20] I. Podlubny, Fractional Differential Equations, vol. 198, Academic Press, San Diego, Calif, USA, 1999.

[21] G. H. Hardy, J. E. Littlewood, and G. Pólya, Inequalities, Cambridge the University Press, Cambridge, UK, 1952.

[22] I. Petráš, "A note on the fractional-order Volta's system," Communications in Nonlinear Science and Numerical Simulation, vol. 15, no. 2, pp. 384-393, 2010. 


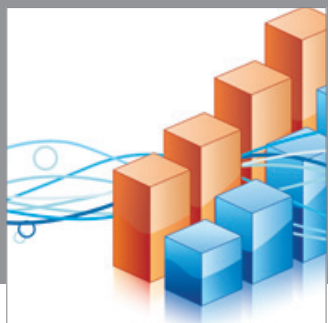

Advances in

Operations Research

mansans

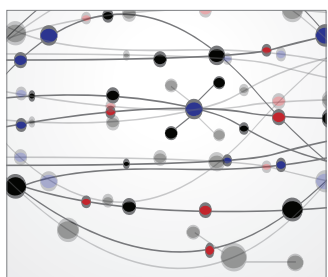

The Scientific World Journal
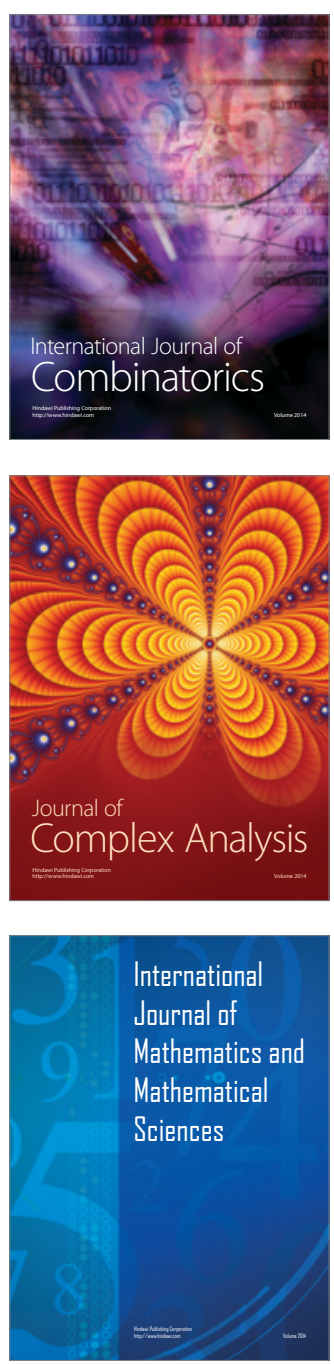
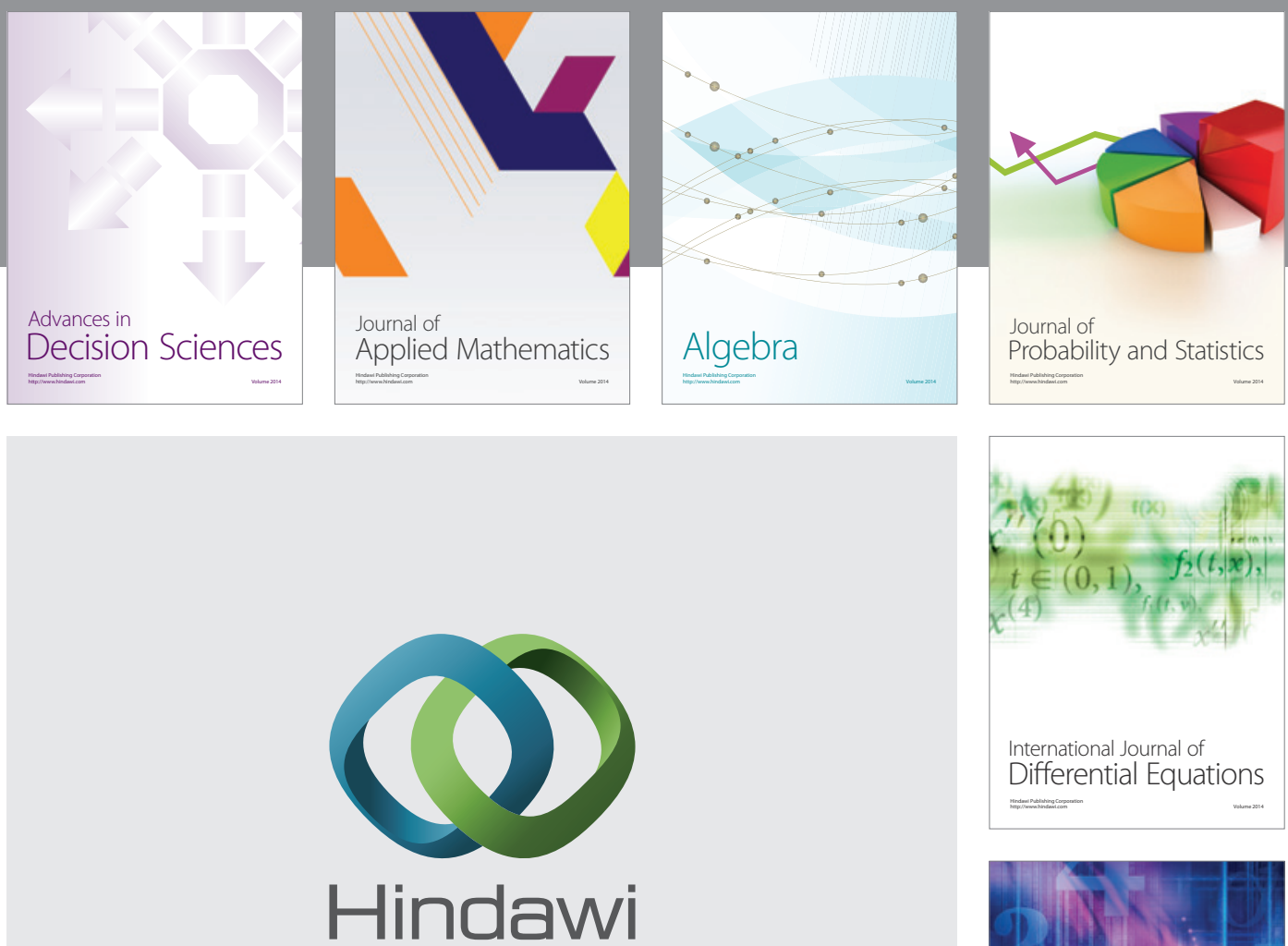

Submit your manuscripts at http://www.hindawi.com
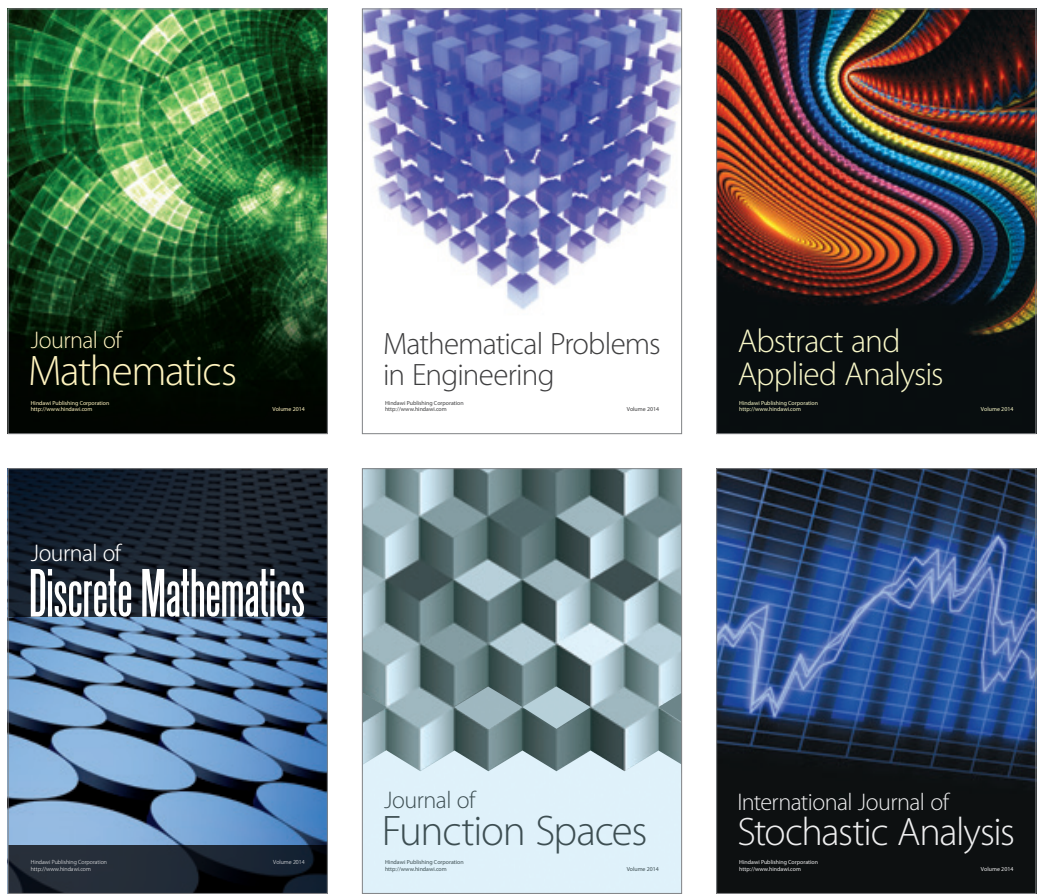

Journal of

Function Spaces

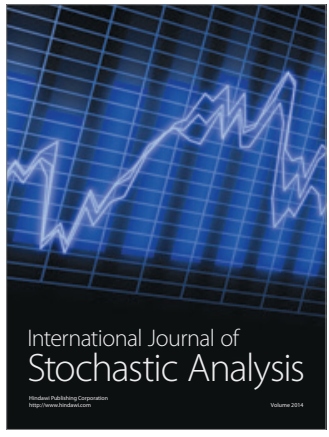

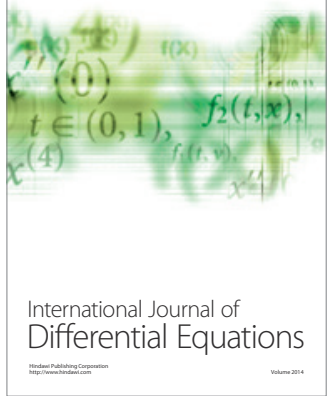
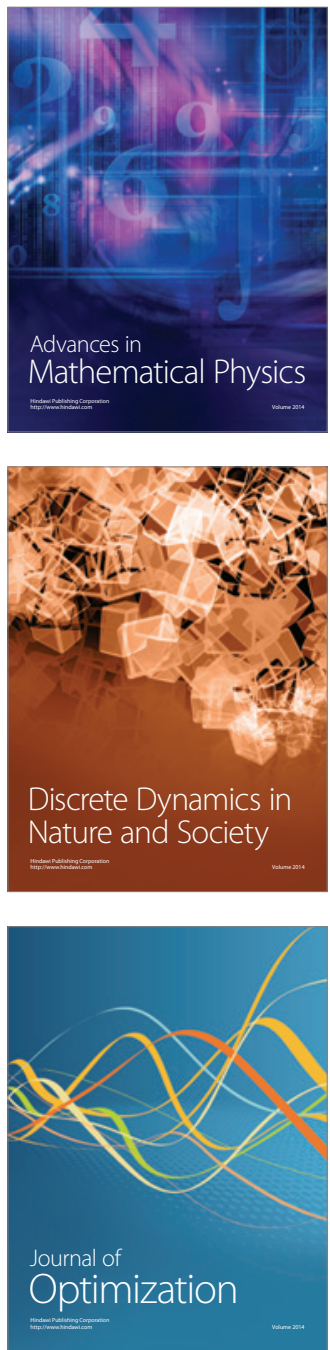